\title{
Review
}

Quan Liang*

\section{Role of transferrin receptor in hepatitis C viral infection}

DOI: $10.2478 / \mathrm{ii}-2018-0016$

Received April 25, 2018; accepted May 04, 2018; published online June 29, 2018

\begin{abstract}
Hepatitis C virus (HCV) is the main pathogen causing chronic hepatitis and primary liver cancer. Various viral proteins and host cell molecules are involved in the HCV cell entry, but the mechanism of infection has not been completely elucidated. The transferrin receptor can act as a receptor for many viruses during cell entry. The transferrin receptor is not only closely related to HCV-induced iron metabolism disorders but also mediates the fusion of HCV with the host cell membrane as a specific receptor for CD81dependent viral adhesion.
\end{abstract}

Keywords: hepatitis C virus, transferrin receptor, cell entry, liver iron overload

Hepatitis $\mathrm{C}$ virus (HCV) is the main pathogen causing chronic hepatitis and even primary liver cancer. HCV virions begin their life cycle through the hepatocyte membrane and enter the cytoplasm. Many viral proteins and host cell molecules are involved in this process [1]. The research progress on the mechanism of HCV cell entry has been slow because of the lack of an effective HCV in vitro cell culture model. Early identification of CD81, low-density lipoprotein receptor, occludin/claudin, scavenger receptor class B type 1, and other host factors may be involved in HCV entry [2]. The determination of the role of HCV envelope glycoproteins in cell entry has also progressed to a certain extent [3]. The establishment of in vitro cell culture systems, such as Huh7, has accelerated the advancement of HCV-related studies, but the mechanism of HCV infection remains unknown.

\section{Structural function of the transferrin receptor (TfR) and regulation mechanism}

Cells uptake iron ions through multiple pathways, of which the transferrin (Tf)/TfR pathway is the main mode for human iron uptake. TfRs include two homologous proteins, namely, receptor for transferrin 1(TfR1) and receptor for transferrin 2 (TfR2) [4]. TfR1, also known as CD71, is widely expressed in human tissues [5], while TfR2 is mainly expressed in hepatocytes, duodenal crypt cells, and red blood cells. The binding force of TfR1 to Tf is 30 times as strong as that of TfR2 to Tf. TfR1 belongs to the type 2 receptor protein and is located at the surface of the cell membrane. It consists of one intracellular region of approximately $15 \mathrm{Ku}$ and an extracellular region with a complex spatial structure. The extracellular region includes a helix domain, a turnhelix region, and a protease-like domain. The formation of a groove of approximately $1 \mathrm{~nm}$ in the protein-like structure is the main site of interaction between TfR1 and Tf. The binding of Tf and iron ions changes their spatial structures accordingly. This process results in the incorporation of iron ions into the protein to form Tf- $\mathrm{Fe}^{2+}$. TfR binds to Tf- $\mathrm{Fe}^{2+}$ at physiological $\mathrm{pH}$, and the Tf-TfR complex is internalized by clathrin-mediated 
endocytosis by pits. The intracellular Tf-TfR complex is transported to endosomal acidification, and the amino acid residue interactions of TfR and Tf cause conformational changes that lead to the release of $\mathrm{Fe}^{2+}$. TfR circulates through the Golgi complex to the cell surface to complete the transport of ferric ion [6].

TfR expression is mainly regulated by intracellular iron ion levels. The $3^{\prime}$-untranslated regions (UTRs) of TfR transcripts play an important role in regulating mRNA stability. This region contains five iron-responsive elements (IREs). The IRE is a set of palindrome sequences, with each sequence consisting of a 30-nucleotide hairpin loop structure. IREs are present at the $3^{\prime}$-UTR and 5'-UTR of the TfR mRNA. The iron regulatory protein (IRP) is involved in the posttranscriptional regulation of TfR expression. This protein can recognize and bind the hairpin loop structure of the UTR IRE of the TfR mRNA and enhance mRNA stability to promote iron uptake by cells [7]. The IRP is divided into IRP1 and IRP2. IRP1 is expressed in all cells, and its structure contains ironsulfur clusters with aconitase activity. At a high intracellular iron level, the affinity of IRP1 and IRE is decreased and TfR mRNA is downregulated. Under iron-deficient conditions, IRP1 is separated from the iron-sulfur clusters and the aconitase activity is lost. The increase in IRE affinity enhances the stability of the TfR mRNA and upregulates the expression of TfR [8,9]. IRP2 lacks the aconitase activity and is not regulated by iron-sulfur clusters. IRP2 binds to the TfR IRE hairpin loop structure to upregulate its expression [10].

\section{TfR and iron metabolism disorders caused by HCV infection}

Liver is an important organ for iron storage and metabolism and also the main target organ for iron overload injury [11]. Iron metabolism disorders are closely related to the worsening of chronic hepatitis C (CHC) conditions and mortality [12]. Saito et al. [13] studied the liver tissues of $\mathrm{CHC}$ patients and healthy controls. The results showed that TfR1 was highly expressed in the liver tissues of most CHC patients than those of healthy controls. However, the TfR1 expression in the hepatic iron overload of CHC patients was not downregulated. These results suggested that TfR1 may be an independent factor for liver iron overload caused by HCV infection. TfR2 mRNA expression was higher in hepatitis C patients than in hepatitis B patients [14]. No significant difference was found in the liver TfR1 mRNA levels between chronic hepatitis B patients and CHC patients, but the TfR2 mRNA levels were significantly increased in CHC patients. This result was positively correlated with the total hepatic iron score. Fujita et al. [15] found that the TfR2 protein level in the liver cells of CHC patients was significantly increased and the iron uptake of hepatocytes through the TfR2 pathway was also enhanced. This phenomenon indicated that elevated TfR2 expression is closely related to HCV infection. In CHC patients receiving interferon antiviral therapy, TfR2 expression was significantly downregulated as HCV viral load decreased, and total hepatic iron score was also decreased. Therefore, HCV infection promotion of TfR2 expression may be an important cause of liver iron overload, and TfR2 may play a more important role in HCV-induced liver iron overload than TfR1.

\section{TfR as a receptor involved in the entry of multiple viruses}

TfR not only participates in the transport of iron ions in cells but also interacts with other protein molecules to perform corresponding biological functions. TfR can act as a receptor for many viruses to enter cells [16]. Moreover, this receptor can also inhibit the nuclear factor $\kappa \mathrm{B}$ signaling pathway by influencing IкB kinase [17]. Radoshitzky et al. [18] found that the Machupo virus (MACV) can infect cells by the interaction of TfR1 with $\alpha$-dystroglycan. Anti-TfR1 antibodies can block MACV cell entry, knockdown TfR1 expression in host cells, and reduce MACV infection rates. Parker et al. [19] found that the entry of canine parvovirus (CPV) was also closely related to TfR1.

CPV can only replicate autonomously during the $S$ phase of cell growth and can be permanently anchored on actively dividing cells by binding to TfR1 [20]. CPV can effectively infect lymphocytes and 
small intestine crypt cells. These phenomena are confirmed by the high expression of TfR1 on the surface of highly fragmented lymphocytes and intestinal crypt cells [21-23]. The mouse mammary tumor virus (MMTV) is a pH-dependent virus. Wang et al. [24] found that this virus can use TfR1 as its receptor to infect cells. The MMTV virions first bind to TfR1 on the cell surface, enter the cell with endocytosis, and undergo $\mathrm{pH}$-dependent membrane fusion in the lysosome. Meanwhile, the arenavirus is one of the sources of infection that causes the viral hemorrhagic renal syndrome in which TfR1 has also been identified as an important receptor protein. In addition, several new world arenaviruses and feline panleukopenia virus (FPV) also use TfR1 as an entrance receptor.

\section{The role of TfR1 in the HCV cell entry}

HCV can infect various cells, such as hepatocytes, peripheral blood mononuclear cells, and T lymphocytes $[25,26]$. HCV-infected cells are the first to bind to target cell surface receptors. Adsorption of HCV on the cell surface mediates virus entry into the cell via receptor molecule complexes. Glycosaminoglycans and lowdensity lipoprotein receptors are involved in virus adhesion, which is mediated by apolipoprotein binding to HCV particles [27]. Subsequently, the virions form a complex with SR-BI and CD81, transport to the tight junction of the cell, and then bind to claudin $1[28,29]$.

HCV-infected host cells may also be dependent on TfR1. Martin and Uprichard [30] found that TfR1 can act as an HCVE1/E2 protein-specific cell receptor during the HCV cell entry. HCV particles efficiently bind to TfR1 without binding to other receptors. This conclusion can also be supported by the direct binding of HCV cell culture to TfR1 on the surface of Chinese hamster ovary cells. Inhibition of TfR1 expression or addition of TfR1 neutralizing antibodies suppresses HCV infection in hepatocytes. Antibody neutralization experiments showed that the 208-212 amino acid residues of TfR1 may be the central region of TfR1 that recognizes HCV. The TfR1 neutralizing antibody had no effect on the repression of HCV infection when the cell surface CD81 molecule was inhibited. This result suggested that TfR1 may mediate viral entry via endocytosis after CD81dependent viral adherence. Further studies have shown that TfR1-mediated HCV entry may depend on the TfR1 trafficking protein (TTP)-related endocytosis mechanism. TTP plays an important role in TfR1-mediated cell phagocytosis. TTP assists TfR1 internalization by clathrin-mediated endocytosis [31]. Inhibition of TTP expression can prevent HCV cell culture infection. FPV also uses TfR1 as a cellular receptor and is involved in clathrin-mediated endocytosis. The FPV capsid protein and Tf can be co-localized with TfR1, enter endocytic vacuole by endocytosis, and then are released into the cytoplasm. If the expression of TfR1 is inhibited, FPV cannot infect cells [32]. However, some viruses, such as MMTV, only use TfR1 as a receptor for cells and do not rely on endocytosis.

\section{Conclusion}

TfR1 is not only involved in the cellular iron ion transport but also mediates the fusion of HCV and host cell membranes as E1/E2 protein-specific receptors after CD81-dependent virus adhesion. This protein plays an important role in HCV cell entry. Therefore, the potential of TfR as an HCV antiviral target deserves attention. Meanwhile, TfR is closely related to the dysregulation of iron metabolism and may be an independent factor for hepatic iron overload caused by HCV infection. A deep understanding of the mechanism of action of TfR in HCV infection will promote the control and treatment of hepatitis C.

Acknowledgments: None.

Conflict of interest: The author states no conflicts of interest. 


\section{References}

[1] Afzal, M. S., Alsaleh, K., Farhat, R., Belouzard, S., Danneels, A., Descamps, V., . . Rouillé, Y. (2015). Regulation of core expression during the hepatitis C virus life cycle. [Q1]. The Journal of General Virology, 96(Pt 2), 311-321

[2] Vercauteren, K., Van Den Eede, N., Mesalam, A. A., Belouzard, S., Catanese, M. T., Bankwitz, D., Meuleman, P. (2014). Successful anti-scavenger receptor class B type I (SR-BI) monoclonal antibody therapy in humanized mice after challenge with HCV variants with in vitro resistance to SR-BI-targeting agents. Hepatology (Baltimore, Md.), 60(5), 1508-1518.

[3] Cocquerel, L., Voisset, C., \& Dubuisson, J. (2006). Hepatitis C virus entry: Potential receptors and their biological functions. The Journal of General Virology, 87(Pt 5), 1075-1084.

[4] Mifuji, R., Kobayashi, Y., Ma, N., Qiang, Q. L., Urawa, N., Horiike, S., Adachi, Y. (2006). Role of transferrin receptor 2 in hepatic accumulation of iron in patients with chronic hepatitis C. Journal of Gastroenterology and Hepatology, 21(1 Pt 1), 144-151.

[5] Chan, K. T., Choi, M. Y., Lai, K. K., Tan, W., Tung, L. N., Lam, H. Y., Law, S. (2014). Overexpression of transferrin receptor CD71 and its tumorigenic properties in esophageal squamous cell carcinoma. Oncology Reports, 31(3), 1296-1304.

[6] Pereira, S. M., Moss, D., Williams, S. R., Murray, P., \& Taylor, A. (2015). Overexpression of the MRI reporter genes ferritin and transferrin receptor affect iron homeostasis and produce limited contrast in mesenchymal stem cells. International Journal of Molecular Sciences,16(7), 15481-15496.

[7] Theil, E. C. (2015). IRE mRNA riboregulators use metabolic iron ( $\mathrm{Fe}(2+))$ to control mRNA activity and iron chemistry in animals. Metallomics, $7(1), 15-24$.

[8] Jiang, X., Wang, H., Shi, W., Shen, Z., Shen, H., \& Li, M. (2014). Hyperinsulinemia induces hepatic iron overload by increasing liver TFR1 via the PI3K/IRP2 pathway. Journal of Molecular Endocrinology, 53(3), 381-392.

[9] Fillebeen, C., Muckenthaler, M., Andriopoulos, B., Bisaillon, M., Mounir, Z., Hentze, M. W., Pantopoulos, K. (2007). Expression of the subgenomic hepatitis C virus replicon alters iron homeostasis in Huh7 cells. Journal of Hepatology, 47(1), $12-22$.

[10] Kühn, L. C. (2015). Iron regulatory proteins and their role in controlling iron metabolism. Metallomics, 7(2), 232-243.

[11] Kohjima, M., Yoshimoto, T., Enjoji, M., Fukushima, N., Fukuizumi, K., Nakamura, T., . . Nakamuta, M. (2015). Hepcidin/ ferroportin expression levels involve efficacy of pegylated-interferon plus ribavirin in hepatitis C virus-infected liver. World Journal of Gastroenterology,21(11), 3291-3299.

[12] Qin, Y., Guo, Y., \& Zhang, Y. (2015). Mechanism, and iron metabolism disorder in patients with Chronic Hepatitis C, J. Clin. Hepatol., 31(2), 291-294.

[13] Saito, H., Fujimoto, Y., Ohtake, T., Suzuki, Y., Sakurai, S., Hosoki, Y., Kohgo, Y. (2005). Up-regulation of transferrin receptor 1 in chronic hepatitis C: Implication in excess hepatic iron accumulation. Hepatology Research, 31(4), 203-210.

[14] Takeo, M., Kobayashi, Y., Fujita, N., Urawa, N., Iwasa, M., Horiike, S., Adachi, Y. (2005). Upregulation of transferrin receptor 2 and ferroportin 1 mRNA in the liver of patients with chronic hepatitis C. Journal of Gastroenterology and Hepatology, 20(4), 562-569.

[15] Fujita, N., Sugimoto, R., Urawa, N., Araki, J., Mifuji, R., Yamamoto, M., Kaito, M. (2007). Hepatic iron accumulation is associated with disease progression and resistance to interferon/ribavirin combination therapy in chronic hepatitis C. Journal of Gastroenterology and Hepatology, 22(11), 1886-1893.

[16] Drakesmith, H., \& Prentice, A. (2008). Viral infection and iron metabolism. Nature Reviews. Microbiology, 6(7), $541-552$.

[17] Tacchini, L., Gammella, E., De Ponti, C., Recalcati, S., \& Cairo, G. (2008). Role of HIF-1 and NF-kappaB transcription factors in the modulation of transferrin receptor by inflammatory and anti-inflammatory signals. The Journal of Biological Chemistry, 283(30), 20674-20686.

[18] Radoshitzky, S. R., Abraham, J., Spiropoulou, C. F., Kuhn, J. H., Nguyen, D., Li, W., Choe, H. (2007). Transferrin receptor 1 is a cellular receptor for New World haemorrhagic fever arenaviruses. Nature, 446(7131), 92-96.

[19] Parker, J. S., Murphy, W. J., Wang, D., O’Brien, S. J., \& Parrish, C. R. (2001). Canine and feline parvoviruses can use human or feline transferrin receptors to bind, enter, and infect cells. Journal of Virology, 75(8), 3896-3902.

[20] Parker, J. S., \& Parrish, C. R. (2000). Cellular uptake and infection by canine parvovirus involves rapid dynamin-regulated clathrin-mediated endocytosis, followed by slower intracellular trafficking. Journal of Virology, 74(4), 1919-1930.

[21] Battilani, M., Balboni, A., Giunti, M., \& Prosperi, S. (2013). Co-infection with feline and canine parvovirus in a cat. Veterinaria Italiana, 49(1), 127-129.

[22] Martella, V., Decaro, N., Elia, G., \& Buonavoglia, C. (2005). Surveillance activity for canine parvovirus in Italy. Journal of Veterinary Medicine. B, Infectious Diseases and Veterinary Public Health, 52(7-8), 312-315.

[23] Moura, I. C., Hermine, O., Lacombe, C., \& Mayeux, P. (2015). Erythropoiesis and transferrin receptors. Current Opinion in Hematology, 22(3), 193-198.

[24] Wang, E., Obeng-Adjei, N., Ying, Q., Meertens, L., Dragic, T., Davey, R. A., \& Ross, S. R. (2008). Mouse mammary tumor virus uses mouse but not human transferrin receptor 1 to reach a low $\mathrm{pH}$ compartment and infect cells. Virology, 381(2), 230-240.

[25] Zhang, M., Zhang, L., Li, H., Chen, Z., Luo, A., Liu, B., . . Hu, P. (2016). Circulating T follicular helper cells are associated with rapid virological response in chronic hepatitis $\mathrm{C}$ patients undergoing peginterferon therapy. International Immunopharmacology, 34, 235-243. 
[26] Waldron P.R., Holodniy M., Peripheral blood mononuclear cell gene expression remains broadly altered years after successful interferon-based hepatitis C virus treatment, J. Immunol. Res., 2015, 2015, 958231, DOI: 10.1155/2015/958231 Epub 2015 Oct 19.

[27] Ma L., Wang P., Jia Z., Research progress of HCV entry related molecule and its mechanism of action, Chin. J. Cell Mol. Imm., 2009, 26(6), 571-573.

[28] Zona, L., Tawar, R. G., Zeisel, M. B., Xiao, F., Schuster, C., Lupberger, J., \& Baumert, T. F. (2014). CD81-receptor associations-Impact for hepatitis C virus entry and antiviral therapies. Viruses, 6(2), 875-892

[29] Labonté, P., Begley, S., Guévin, C., Asselin, M. C., Nassoury, N., Mayer, G., Seidah, N. G. (2009). PCSK9 impedes hepatitis C virus infection in vitro and modulates liver CD81 expression. Hepatology (Baltimore, Md.), 50(1), 17-24.

[30] Martin D.N., Uprichard S.L., Identification of transferrin receptor 1 as a hepatitis C virus entry factor, Proc. Natl. Acad. Sci. USA, 2013, 110(26), 10777-10782.

[31] Tosoni, D., Puri, C., Confalonieri, S., Salcini, A. E., De Camilli, P., Tacchetti, C., \& Di Fiore, P. P. (2005). TTP specifically regulates the internalization of the transferrin receptor. Cell, 123(5), 875-888.

[32] Truyen U., Parrish C.R., Feline panleukopenia virus: its interesting evolution and current problems in immunoprophylaxis against a serious pathogen, Vet. Microbiol., 2013, 165(1/2), 29-32. 